\title{
The mechanism of variegation in immutans provides insight into chloroplast biogenesis
}

\author{
Andrew Foudree, Aarthi Putarjunan, Sekhar Kambakam, Trevor Nolan, Jenna Fussell, Gennady Pogorelko \\ and Steve Rodermel*
}

Department of Genetics, Development, and Cell Biology, lowa State University, Ames, IA, USA

\author{
Edited by: \\ Tatjana Kleine, \\ Ludwig-Maximilians-Universität \\ München, Germany \\ Reviewed by: \\ Toshiharu Shikanai, Kyoto University, \\ Japan \\ Ko Noguchi, The University of Tokyo, \\ Japan \\ *Correspondence: \\ Steve Rodermel, Department of \\ Genetics, Development, and Cell \\ Biology, lowa State University, 457 \\ Bessey Hall, Ames, IA 50011, USA. \\ e-mail: rodermel@iastate.edu
}

\begin{abstract}
The immutans (im) variegation mutant of Arabidopsis has green and white-sectored leaves due to the absence of fully functional plastid terminal oxidase (PTOX), a plastoquinol oxidase in thylakoid membranes. PTOX appears to be at the nexus of a growing number of biochemical pathways in the plastid, including carotenoid biosynthesis, PSI cyclic electron flow, and chlororespiration. During the early steps of chloroplast biogenesis, PTOX serves as an alternate electron sink and is a prime determinant of the redox poise of the developing photosynthetic apparatus. Whereas a lack of PTOX causes the formation of photooxidized plastids in the white sectors of im, compensating mechanisms allow the green sectors to escape the effects of the mutation. This manuscript provides an update on PTOX, the mechanism of im variegation, and findings about im compensatory mechanisms.
\end{abstract}

Keywords: IMMUTANS, PTOX, variegation, chloroplast, photosynthesis, carotenoids, chloroplast biogenesis, retrograde signaling

\section{INTRODUCTION}

Variegation mutants provide an excellent system to explore the mechanisms of chloroplast biogenesis and the nature of communication between the nucleus-cytoplasm, chloroplast, and mitochondrion (Rodermel, 2001; Yu et al., 2007). The leaves of these mutants contain green and chlorotic (white, yellow) sectors that arise as a consequence of mutations in nuclear or organelle genes (Tilney-Bassett, 1975). Whereas cells in the green sectors have morphologically normal chloroplasts, cells in the chlorotic sectors contain pigment-deficient plastids with abnormal membrane structures (Aluru et al., 2001). One common mechanism of variegation involves the induction of defective chloroplasts (or mitochondria) by mutations in nuclear genes that code for organelle proteins. In some cases the green and white cells have different genotypes. For example, transposable element activity can inactivate a gene required for normal chloroplast function in some cells (chlorotic cells) while excision can reconstitute gene function (green cells). In other cases of variegation, the two types of cells have the same (mutant) genotype, indicating that the gene product is required in some, but not all cells of the mutant. This raises the question of how the green cells escape this requirement. Is there a compensating activity? Our operating hypothesis is that answers to this question will provide insight into mechanisms of variegation, as well as into pathways and regulatory networks of chloroplast biogenesis.

The past decade has witnessed a growing interest in the use of variegation mutants to address fundamental questions in photosynthesis and chloroplast biogenesis (Yu et al., 2007; Liu et al., 2010). Yet, mechanisms of variegation are still poorly understood. Here we focus on the mechanism of variegation in immutans (im), which defines the gene for plastid terminal oxidase (PTOX), a versatile quinol oxidase in plastid membranes. Early studies led to the notion that this mechanism involves the attainment of a threshold by developing plastids (the threshold hypothesis). In this manuscript, we review the underlying assumptions and experiments in support of this hypothesis and discuss approaches and studies that have been used to gain insight into factors that compensate for a lack of PTOX.

\section{IMMUTANS}

The im variegation mutant is one of the oldest Arabidopsis mutants, reported independently by Rédei and Röbbelen in the 1960's (Rédei, 1963; Röbbelen, 1968). Cells in the green sectors of im have morphologically normal chloroplasts, whereas cells in the white sectors are heteroplastidic and contain abnormal plastids that lack pigments and organized lamellae, as well as rare, normalappearing chloroplasts (Wetzel et al., 1994). The extent of white sector formation in im is promoted by high light and low temperature (Rédei, 1963, 1967; Röbbelen, 1968; Wetzel et al., 1994; Rosso et al., 2009). HPLC analyses showed that the white sectors accumulate the colorless $\mathrm{C}_{40}$ carotenoid intermediate, phytoene, indicating that im is impaired in the activity of phytoene desaturase (PDS), the enzyme which converts phytoene to zeta-carotene (Wetzel et al., 1994). All the steps of carotenogenesis take place in the plastid and are mediated by nuclear-encoded enzymes that are imported into the organelle post-translationally, and PDS mediates an early step of the pathway (DellaPenna and Pogson, 2006). An inhibition of PDS activity would thus result in lack of accumulation of downstream, colored (photoprotective) carotenoids, and under high light conditions, would be anticipated to give rise to white, photooxidized plastids. A defect of this sort is consistent with the light-sensitivity of $\mathrm{im}$; as in other carotenoid mutants, the higher the light intensity, the greater the extent of photooxidation (white sector formation) (Oelmüller, 1989; Mayfield and Taylor, 2005). The presence of normal-appearing chloroplasts in im, on the other hand, suggests that some plastids are able to bypass the 
requirement for the $I M$ gene product, likely because of compensating activities that make them less susceptible to photooxidation early in their development.

\section{PTOX FUNCTION}

IMMUTANS was cloned in Arabidopsis by both map-based methods (Wu et al., 1999) and T-DNA tagging (Carol et al., 1999). The gene product (IM) was discovered to be a plastid membrane protein that is distantly related ( $37 \%$ amino acid similarity) to alternative oxidase (AOX), a mitochondrial inner membrane protein which functions in the alternative (cyanide-resistant) pathway of respiration, where it transfers electrons from ubiquinol to molecular oxygen (Wu et al., 1999). Central among its physiological functions, AOX is an important sensor of cellular redox balance (Giraud et al., 2008; McDonald, 2008). Similar to AOX, IM has quinol oxidase activity in vivo and in vitro and, consequently, it has been designated PTOX (Joët et al., 2002; Josse et al., 2003). PTOX is found in some cyanophages, which might serve as vectors for transfer of PTOX among cyanobacteria, but otherwise appears to be limited to oxygenic photosynthetic prokaryotes and eukaryotes, where it is found in all lineages (McDonald et al., 2011). IM is generally present as a single copy gene, although two copies are found in some cyanobacteria, red algae and green algae (Wang et al., 2009; Houille-Vernes et al., 2011). In chloroplasts, PTOX is bound to the stromal lamellae of thylakoids and is modeled as an interfacial membrane protein whose active site faces the stroma (Berthold et al., 2000; Joët et al., 2002; Lennon et al., 2003). It does not appear to be present in chloroplast envelope membranes.

Several functions have been ascribed to PTOX. The phytoeneaccumulation phenotype of im led to the suggestion that PTOX serves as the terminal oxidase of an oxygen-dependent redox pathway that desaturates phytoene (Beyer et al., 1989; Mayer et al., 1990, 1992; Hugueney et al., 1992; Schulz et al., 1993; Nievelstein et al., 1995; Norris et al., 1995; Al-Babili et al., 1996). This pathway is thought to involve transfer of electrons from phytoene to plastoquinone (PQ) via PDS, forming $\zeta$-carotene and plastoquinol $\left(\mathrm{PQH}_{2}\right)$, and from $\mathrm{PQH}_{2}$ to molecular oxygen via PTOX, forming water and PQ (Carol et al., 1999; Wu et al., 1999; Rosso et al., 2009). Thylakoids of developing im plastids have over-reduced PQ pools (Rosso et al., 2009), and according to this pathway, the accumulation of phytoene in im can be explained by a decreased supply of PQ available to PDS, as suggested by Okegawa et al. (2010), and/or because electron transfer from phytoene into an over-reduced PQ pool is not energetically favorable (Rochaix, 2011). In addition to the phytoene-accumulation phenotype of mutants that lack PTOX (im in Arabidopsis and the orthologous ghost mutant in tomato) (Wetzel et al., 1994; Josse et al., 2000; Barr et al., 2004; Shahbazi et al., 2007), an involvement of PTOX in carotenogenesis is suggested by the close coordination between PTOX expression and carotenoid production in a number of systems, perhaps most strikingly in chromoplasts during the ripening of tomato, citrus, and pepper fruit (Josse et al., 2000; Barr et al., 2004). Another noteworthy example is the upregulation of PTOX expression in etiolated seedlings of Arabidopsis treated with paclobutrazol (PAC), an inhibitor of gibberellin biosynthesis; PAC causes an increase in carotenoid production (Rodríguez-Villalón et al., 2009).
It might be noted that all the enzymes of carotenogenesis are present in chloroplast envelopes, with the exception of PDS which is also found in thylakoids (Joyard et al., 2009). The involvement of PTOX in carotenogenesis is therefore puzzling given its apparent exclusive location in thylakoids (Lennon et al., 2003). This suggests that carotenoid intermediates are trafficked between the envelope and thylakoids, perhaps via vesicles, by connections between the envelope and thylakoids, or by carrier proteins (Adam et al., 2011; Ngaki et al., 2012). There might also be PTOX-independent mechanisms of plastoquinol oxidation in envelopes, such as $\mathrm{PQH}_{2}$ autooxidation (Khorobrykh and Ivanov, 2002), the activity of another plastoquinol oxidase (Buchel and Garab, 1995; Lajko et al., 1997; Joët et al., 2002), or perhaps a plastoquinol peroxidase (Casano et al., 2000; Rumeau et al., 2007).

IM mRNAs are ubiquitously expressed in Arabidopsis, and the anatomies of various plastid types are affected in im (Aluru et al., 2001), as well as in tomato ghost (Josse et al., 2000; Barr et al., 2004). However, a positive correlation between IM mRNA levels and carotenoid accumulation does not always hold, and $I M$ is expressed highly in some tissues with low carotenoid accumulation (Aluru et al., 2001). Considered together, these observations argue that the role of PTOX in plastid metabolism is not limited to carotenogenesis. In fact, since its discovery over 13 years ago, it has become apparent that PTOX resides at the nexus of a growing number of redox pathways in the plastid. For instance, in vitro and in vivo studies have demonstrated that PTOX is the terminal oxidase of chlororespiration (non-photochemical reduction of the PQ pool) (Peltier and Cournac, 2002; Rumeau et al., 2007). Chlororespiration involves the transfer of electrons from $\mathrm{NAD}(\mathrm{P}) \mathrm{H}$ (and/or Ferredoxin) to PQ via a thylakoid $\mathrm{NAD}(\mathrm{P}) \mathrm{H}$ dehydrogenase (NDH) complex, and from $\mathrm{PQH}_{2}$ to molecular oxygen via PTOX (Peng et al., 2012). One purpose of chlororespiration is to help poise the redox state of the PQ pool during cyclic electron flow (CEF) around PSI (Trouillard et al., 2012). In addition to chlororespiration, PTOX mediates chromorespiration, the analogous process in chromoplasts (Josse et al., 2000; Barr et al., 2004; Rodrigo et al., 2004; Shahbazi et al., 2007).

Safety valves are photoprotective mechanisms that dissipate excess photons and electrons (Niyogi, 2000). They include nonphotochemical quenching mechanisms and alternative electron acceptors that prevent over-reduction of photosynthetic electron carriers, thereby protecting PSI and PSII from photodamage (Aro et al., 1993; Allahverdiyeva et al., 2005). Soon after its discovery, Niyogi (2000) proposed that PTOX-mediated electron flow from $\mathrm{PQH}_{2}$ to $\mathrm{O}_{2}$ might act as a safety valve. Early support for this hypothesis came from studies showing that the green leaf sectors of im have morphological, biochemical, and molecular adaptations similar to plants acclimated to growth in high light, even when grown in permissive (low light) conditions (Aluru et al., 2001). Early studies also showed that IM mRNAs and proteins are significantly upregulated in high light conditions in antisense mutants of tobacco that lack both ascorbate peroxidase and catalase (Rizhsky et al., 2002). A number of experiments have since supported the safety valve hypothesis in systems as diverse as the alpine species Ranunculus glacialis and Soldanella alpina (Streb et al., 2005; Laureau et al., 2011); oat exposed to heat and high light (Quiles, 2006); mature green leaves and green fruit of the tomato ghost mutant 
subjected to high light stress (Shahbazi et al., 2007); wild species of Brassica fruticulosa versus the agricultural species Brassica oleracea adapted to both heat and high light (Díaz et al., 2007); the salt-stressed halophyte Thellungiella (Stepien and Johnson, 2009); and cold-acclimated Lodgepole pine (Savitch et al., 2010).

In addition to altered expression in response to environmental factors, PTOX is upregulated in various photosynthetic mutants, including the gun4 mutant of Chlamydomonas and the tobacco $r b c L$ and $p s b A$ deletion mutants. The gun4 mutants lack GUN4, a regulatory subunit of $\mathrm{Mg}$-chelatase, and enhanced PTOX activities in these mutants were suggested to play a physiological role in decreasing PSII excitation pressures (Formighieri et al., 2012). The $r b c L$ mutants lack Rubisco and consequently two major electron sinks, $\mathrm{CO}_{2}$ fixation and photorespiration; it was proposed that elevated PTOX levels in these mutants reduce oxidative pressure on both PSI and PSII (Allahverdiyeva et al., 2005). In the $p s b A$ mutants, which lack the D1 protein of PSII, it was hypothesized that enhanced PTOX levels primarily support increased carotenoid production as a way to quench singlet oxygen generated by free LHCII accumulation in the mutant (Baena-González et al., 2003). Despite the growing number of examples of PTOX upregulation, cis and trans regulatory factors have yet to be reported.

The demonstration that PTOX serves as a safety valve in a particular system is not straightforward and must rely on more than gene expression data, since there are many ways of preventing overreduction of the electron transport chain, such as upregulation of downstream electron sinks, as found in some cold-tolerant plants, or upregulation of the Mehler reaction (e.g., Gray et al., 1997; Savitch et al., 2000); alterations in PTOX expression might be a secondary effect. There are also some cases where PTOX does not act as a safety valve. For example, overexpression of Arabidopsis PTOX does not impart increased resistance to photoinhibition in mature tobacco leaves (Joët et al., 2002), nor does it regulate the redox state of the PQ pool during steady state photosynthesis in Arabidopsis (Rosso et al., 2006). These findings are consistent with the observation that the steady state flux of electrons through PTOX is relatively minor - estimated to be $<2 \%$ of the flux through the photosynthetic electron transport chain (PETC) (Ort and Baker, 2002). On the other hand, these same studies showed that PTOX is important in preventing over-reduction of PSII acceptors during dark to light transients (Joët et al., 2002), and that it protects against PSI photoinhibition (Rosso et al., 2006). The latter has been confirmed in recent experiments showing that PTOX acts as a safety valve in Arabidopsis under stress conditions of low temperature and high light, where it protects against both PSII and PSI photoinhibition (Ivanov et al., 2012).

In this context it is important to note that in contrast to PSII, where there are a number of photochemical and nonphotochemical mechanisms to avoid over-reduction of PSII acceptors, the repertoire is rather more limited for PSI and includes, most prominently, the water-water cycle (reviewed in Rumeau et al., 2007). PTOX might thus turn out to be an important mechanism to reduce electron pressure on PSI, as during stress when $\mathrm{NADPH} / \mathrm{ATP}$ ratios are high and CEF around PSI is activated; in concert with NDH, PTOX might serve to poise the redox state of intersystem carriers by recycling electrons to PQ. This might also be the case during chloroplast biogenesis when it has been demonstrated that PTOX is a central regulator of thylakoid redox and PSII excitation pressure (Rosso et al., 2009).

Although not within the scope of this review, it might be mentioned that the functions described for PTOX in cyanobacteria and eukaryotic algae are similar to those in higher plants (reviewed by McDonald et al., 2011). These include carotenogenesis and response to diverse environmental stresses, such as high light, temperature, salt, iron, and phosphate deprivation (Moseley et al., 2006; Bailey et al., 2008; Wang et al., 2009). One difference is that cyanobacterial respiratory and PETCs share some components, which offers the possibility of PTOX control of both activities.

In summary, there is growing evidence that PTOX is a versatile terminal oxidase in chloroplast metabolism and that its primary physiological role varies from taxon to taxon. This might not be surprising, since PTOX is only one element of a large network of factors involved in stress tolerance, pigment biosynthesis, and photosynthetic control (regulation of ATP/NADPH ratios) (Kramer and Evans, 2011; Foyer et al., 2012). In such a network, it might be anticipated that the relative importance of a given element varies according to developmental and/or physiological context. For instance, PTOX might turn out to play a more significant regulatory role in PSI cyclic electron transport in bundle sheath cells of C4 plants than in mesophyll cells of C3 plants; in contrast to C3 plants, cyclic electron transport is a key regulator of ATP synthesis during C4 photosynthesis (Rumeau et al., 2007; Foyer et al., 2012). Given the ubiquity of PTOX expression, it might also be predicted that novel functions will be found for PTOX, especially in non-green plastids. A schematic diagram of PTOX in thylakoids is shown in Figure 1.

\section{MECHANISM OF VARIEGATION: THE THRESHOLD HYPOTHESIS \\ STUDIES USING MATURE LEAVES}

Nearly all molecular and biochemical studies on im have been conducted using fully expanded rosette leaves; for comparative studies, green versus white sectors have been obtained by dissection or via fluorescence-activated cell sorting (FACS) (Wetzel et al., 1994; Meehan et al., 1996; Aluru et al., 2009). Alternatively, allgreen $i m$ leaves have been produced by altering light conditions during early seedling development (Rosso et al., 2009).

The notion that im variegation is due to a threshold came from two sets of observations. The first was that the white sectors of $i m$ are heteroplastidic, indicating that im behaves in a plastid autonomous manner, i.e., plastid phenotypes within a cell are determined independently in the im background. The second was that intermediate plastid phenotypes are not seen; plastids in the green sectors look similar to one another, as do abnormal plastids in the white sectors (Figure 2). Taken together with the finding that the white (but not green) sectors accumulate phytoene, the first iteration of the threshold hypothesis held that plastid phenotype in $i m$ is determined by the attainment of a threshold of PDS activity during chloroplast biogenesis: developing plastids with abovethreshold activities are able to produce enough downstream, photoprotective carotenoids to form normal-appearing chloroplasts (green sectors), whereas plastids with below-threshold PDS activities are deficient in colored carotenoid production and susceptible to photooxidation (white plastids and sectors) (Wetzel et al., 1994; 


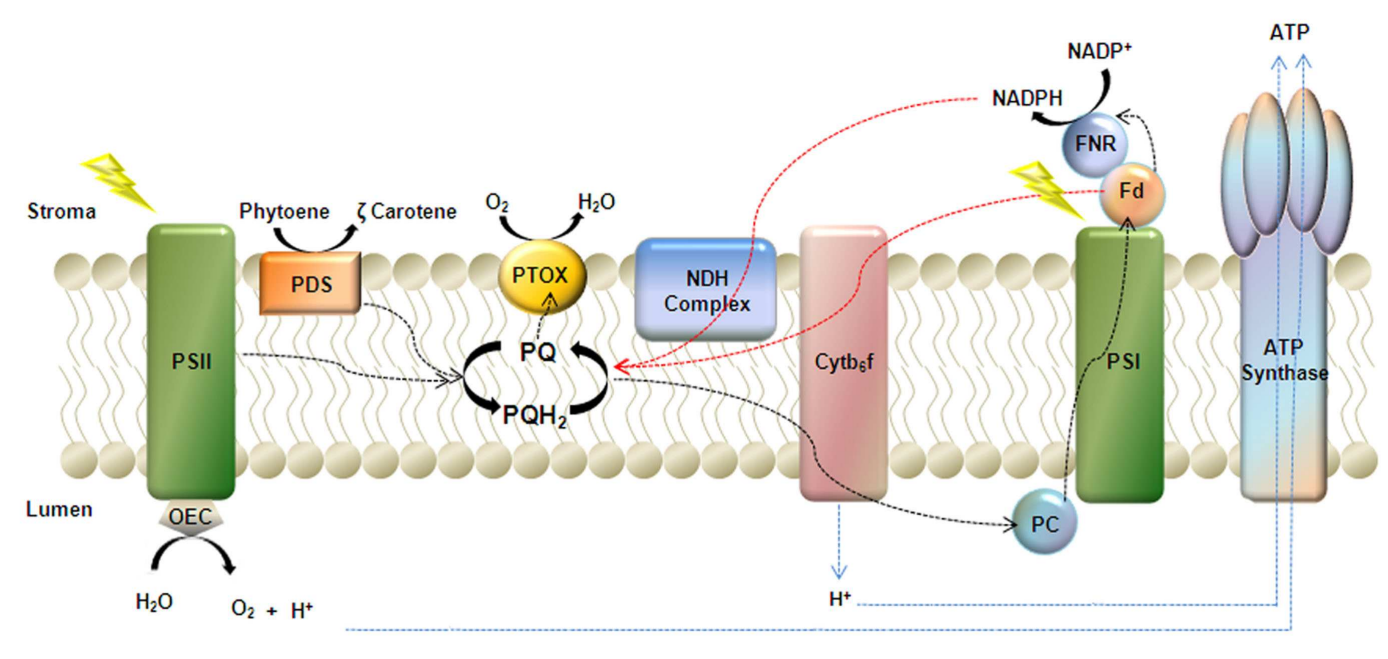

Linear Electron Flow $\longleftarrow$
Cyclic Electron Flow
Proton Flow

FIGURE 1 | Model of PTOX as a cofactor of phytoene desaturase

(PDS). PTOX is a plastoquinol terminal oxidase that regulates the redox state of the plastoquinone pool (PQ) during early chloroplast biogenesis. Electrons from linear electron flow, cyclic electron flow mediated by either NDH or the Fd dependent PGR5 pathway, and the desaturation of phytoene, feed into the PO pool. PTOX plays a pivotal role in transferring electrons from the $\mathrm{PQ}$ pool to molecular oxygen thus keeping the pool oxidized.

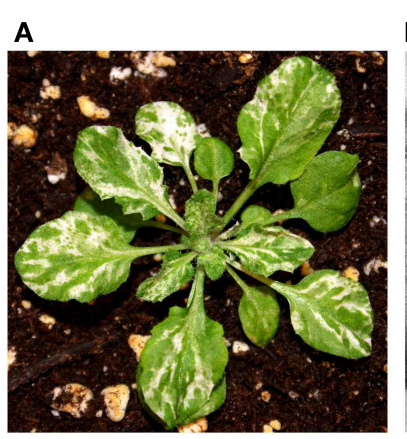

\section{B}

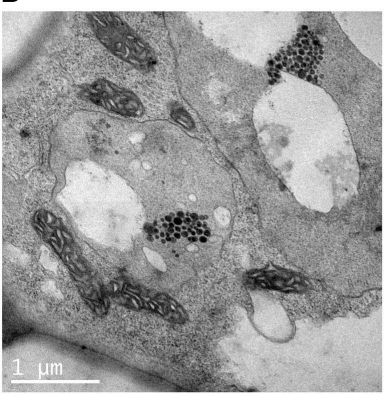

C

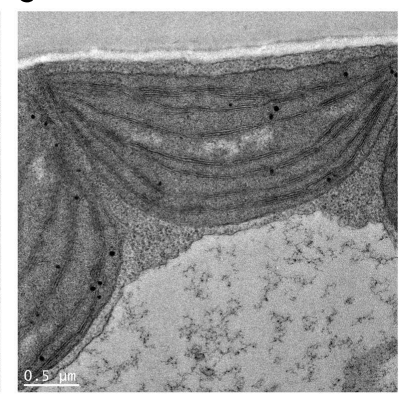

FIGURE 2 | Ultrastructural analysis of immutans (A). A representative immutans plant grown at $22^{\circ} \mathrm{C}$ under continuous illumination for 7 days at $5 \mu \mathrm{mol}$ photons $\mathrm{m}^{-2} \mathrm{~s}^{-1}$ followed by 3 weeks at $100 \mu \mathrm{mol}$ photons $\mathrm{m}^{-2} \mathrm{~s}^{-1}$.
(B) Representative plastids from an im white sector. The plastids lack organized lamellar structures and contain vacuoles and numerous plastoglobules. (C) Representative chloroplast from an im green sector.
Wetzel and Rodermel, 1998). This hypothesis was later refined after $I M$ was cloned and its function was defined (described in greater detail later).

Chloroplast biogenesis involves the differentiation of a small number of proplastids in the shoot apical meristem into chloroplasts early in leaf primordial development (Mullet, 1988). This process is accompanied by several rounds of plastid division (Robertson et al., 1995; Pyke and López-Juez, 1999). In C3 plants like Arabidopsis, leaf expansion along the proximal-distal axis results from cell divisions at the base of the leaf, while cell divisions at the center of the leaf and extending outward cause lateral expansion (Pyke et al., 1991; Van Lijsebettens and Clarke, 1998). Hence, the oldest chloroplasts are present in cells at the tips and margins of the leaf, whereas the youngest ones are found in cells at the base of the leaf and at the midveins. Although plastid number depends on cell size (Possingham and Lawrence, 1983), a typical Arabidopsis meristem cell contains a dozen or so proplastids whereas a mesophyll cell contains from 100 to 120 mature chloroplasts (Mullet, 1988; Pyke and López-Juez, 1999). The numbers of plastids in im green and white sectors have not yet been reported.

Wetzel et al. (1994) performed light-shift experiments to determine when PTOX activity is first required following germination. These studies showed that cotyledon pigmentation (all-green, allwhite, or variegated) is influenced by the intensity of light (low or high) perceived between 0 and $24 \mathrm{~h}$ after seed coat breakage; the intensity perceived before or after this interval did not matter. During this period, proplastids differentiate into chloroplasts within the developing cotyledon (Mansfield and Briarty, 1996). 
This indicates that PTOX plays a crucial role in early chloroplast biogenesis.

We have recently exploited confocal microscopy to visualize im variegation during leaf primordia development. Figure 3 shows the red autofluorescence from chloroplasts in cells of wild type and $i m$ leaf primordia; each primordium is located between the two cotyledons. The figure dramatically illustrates that im cotyledons and primordia have fewer chloroplasts than wild type, and that im variegation develops very early in leaf development.

\section{STUDIES USING GREENING AS A MODEL SYSTEM}

Etioplasts in dark-grown angiosperm seedlings have prolamellar bodies (PLB) that contain abundant ternary complexes of protochlorophyllide (PChlide), PChlide reductase (POR), and NADPH (Solymosi and Schoefs, 2010). Upon exposure to light, PChlide is converted to chlorophyllide (Chlide) and the membranes of the PLB disperse to form thylakoids and organized grana (Adam et al., 2011). Most of the protein components of the photosynthetic apparatus are present in etioplasts, and hence de-etiolation (greening) primarily involves the coordinated synthesis and assembly of pigments and pigment-binding proteins to form functional electron transport chains (von Zychlinski et al., 2005; Blomqvist et al., 2008; Kanervo et al., 2008). Photosynthetic competence is attained in Arabidopsis $\sim 5 \mathrm{~h}$ after light induction at $22^{\circ} \mathrm{C}$ and $100 \mu \mathrm{mol}$ photons $\mathrm{m}^{-2} \mathrm{~s}^{-1}$ (Rosso et al., 2009).

Using Arabidopsis de-etiolation as a model system, Hüner's group found that white sector formation in im is positively correlated with elevated excitation pressures during early greening, irrespective of whether excitation pressures are modulated by light and/or temperature (Rosso et al., 2009). Excitation pressure is a relative measure of the reduction state of $Q_{\mathrm{A}}$, the first stable electron acceptor of PSII (Dietz et al., 1985; Hüner et al., 1998), and is measured by chlorophyll $a$ fluorometry (Krause and Weis, 1991). The elevated excitation pressures in im were accompanied by delayed biogenesis of the thylakoid membrane, as monitored by the abundance of protein markers of chloroplast development (Rosso et al.,

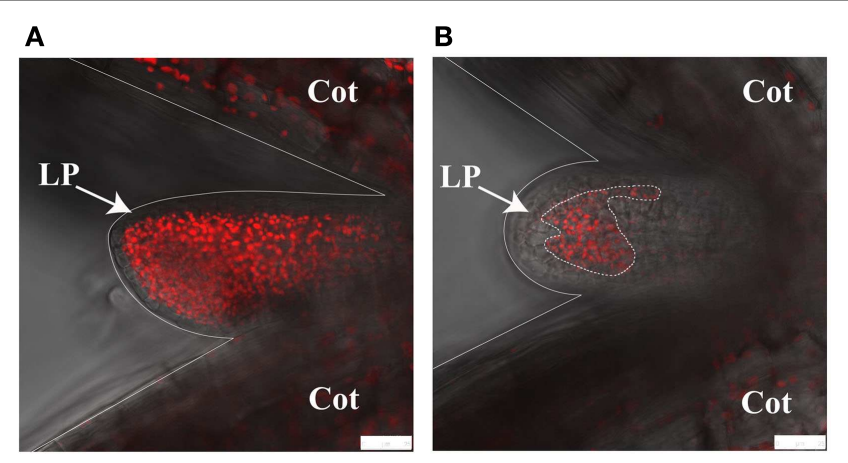

FIGURE 3 | Confocal microscopy of leaf primordia. The images show first leaf primordia (LP) and flanking cotyledons (Cot) from wild type (A) and immutans (B). Images were captured by taking a confocal image of autofluorescence (red) and overlaying it with a non-confocal transmitted light image (gray). Plants were grown at $22^{\circ} \mathrm{C}$ under continuous illumination for 3 days at $5 \mu \mathrm{mol}$ photons $\mathrm{m}^{-2} \mathrm{~s}^{-1}$ followed by 2 days at a light intensity $100 \mu \mathrm{mol}$ photons $\mathrm{m}^{-2} \mathrm{~s}^{-1}$. White scale bars represent $25 \mu \mathrm{m}$.
2009). Given these considerations, our current working model of im variegation assumes that above-threshold excitation pressures predispose developing plastids to photooxidation, whereas im plastids with below-threshold excitation pressures have the capacity to develop into normal chloroplasts. This model is based on the twin notions of plastid autonomy and plastid heterogeneity. We surmise that excitation pressures vary from plastid-to-plastid in the developing leaf primordium because of intrinsic differences in plastid biochemistry caused, for example, by gradients in the leaf, sometimes steep, of light and of determinants of light capture, and use (Smith et al., 1997), including cell-specific circadian rhythms that dictate reactive oxygen species (ROS) scavenging capacities (Velez-Ramirez et al., 2011). Such heterogeneity appears to be widespread and has recently been reported in species as diverse as trees (Solymosi et al., 2012). One advantage of this model is that it can account for the phytoene-accumulation phenotype of $i m$, as well as for the ultrastructural defects of im white plastids. For instance, one of the primary targets of over-reduction might be membrane biogenesis since a prominent effect of ROS formation is lipid peroxidation of polyunsaturated fatty acids (Møller et al., 2007). A lack of plastid galactolipids coupled with a lack of stabilizing carotenoids might impede membrane formation, giving rise to the large vesicles seen in im white plastids (Figure 2).

In summary, our current model of im variegation proposes that the pattern of green/white sectoring is a reflection of the pattern of leaf development in C3 plants, coupled with a heterogeneity in plastid-to-plastid excitation pressure during early cell and plastid development, i.e., the chaotic sectoring in the mature leaf is established in the leaf primordium. Recent support for the threshold hypothesis has come from the altered APX2 expression 13 (alx13) variegation mutant of Arabidopsis (Woo et al., 2011).

\section{COMPENSATING FACTORS}

Because the green sectors of im contain morphologically normal chloroplasts, we have proposed that they arise from plastids that escape the effects of the $\mathrm{im} / \mathrm{im}$ mutation early in chloroplast biogenesis, presumably by the action of compensating factors that affect excitation pressure thresholds, directly or indirectly. Two approaches have been used to gain insight into these factors: (1) molecular characterization of green versus white im sectors; and (2) identification and characterization of second-site suppressors of $i m$ variegation. These approaches will be summarized briefly.

\section{HOW DO THE GREEN AND WHITE SECTORS OF im DIFFER?}

The green leaf sectors of $i m$ have higher photosynthetic rates than wild type leaves, monitored either by rates of $\mathrm{O}_{2}$ evolution or ${ }^{14} \mathrm{CO}_{2}$ uptake (Aluru et al., 2001, 2007). They also have elevated chl $a / b$ ratios and enhanced chlorophyll amounts; FACS analyses have revealed these increases are due to elevated chloroplast numbers (per unit volume) rather than to enhanced amounts of chlorophyll per chloroplast (Meehan et al., 1996). Accompanying these changes, the anatomies of the green leaf sectors are reminiscent of leaves adapted to growth in high light conditions, e.g., they are thicker than normal due to enlarged air spaces, mesophyll cells, and epidermal cells (Aluru et al., 2001). On the other hand, the white leaf sectors have a normal thickness, but their palisade cells fail to expand. This perturbation is consistent with an impairment 
in retrograde signaling that regulates leaf developmental programming (Rodermel, 2001; López-Juez, 2009; Ruckle and Larkin, 2009). We have made a similar proposal to explain reduced fruit size and disruptions in pericarp tissue morphogenesis in the tomato ghost mutant (Barr et al., 2004).

Consistent with elevated rates of photosynthesis, the green leaf sectors have increased Rubisco and sucrose phosphate synthase (SPS) activities, enhanced starch and sucrose pool sizes, and an altered pattern of carbohydrate partitioning that favors sucrose over starch (Aluru et al., 2007). By contrast, the white sectors accumulate low levels of sucrose and have increased acid invertase activities. These observations suggest that sucrose moves along a gradient from the green to the white cells, where it is hydrolyzed and used for growth. It is therefore possible that photosynthetic rates in the green sectors are controlled, in part, by sink demand, and that the elevation of photosynthetic rates in the green sectors is part of a growth strategy to compensate for reductions in total source tissue (Aluru et al., 2007).

Global transcriptomics experiments have revealed that some of the differences between the green and white im sectors are likely due to alterations in transcript abundance (Aluru et al., 2009). In particular, genes for photosynthesis and photosynthesis-related processes are repressed in im white tissues, while genes for sucrose catabolism, transport, mitochondrial electron transport, and fermentation are induced. This suggests that energy is derived via aerobic and anaerobic metabolism of imported sugar in the im white cells, in accord with findings from the biochemical studies, discussed above. The profiling studies also showed that oxidative stress response genes are generally induced in both the green and white im tissues, but that some stress genes are significantly more upregulated in the green than white sectors. These genes are targets for investigation as potential compensating factors.

\section{SECOND-SITE SUPPRESSORS OF im VARIEGATION}

The above studies have been conducted with mature leaves, and although they have provided valuable information about $i m$, they are limited in their usefulness regarding information about the primary lesion in im because they describe developmental, physiological, and biochemical states that represent long term adaptations to the lack of PTOX in the leaf. They do not provide information about the primary alterations that occur when the mutation first becomes active during chloroplast biogenesis and when compensating mechanisms might first come into play.

Given that all alleles of $i m$ isolated to date are null (Carol et al., 1999; Wu et al., 1999), an attractive strategy to identify compensating factors is to take advantage of Arabidopsis genetics to characterize second-site suppressors of $i m$ variegation, i.e., second-site mutations that reverse the im defect, generating all-green plants. These factors would be anticipated to define elements and/or processes that are able to substitute for or bypass the requirement for PTOX activity during chloroplast biogenesis. One advantage of using a genetic approach to identify these factors is that the early stages of chloroplast development are difficult to access by other approaches (biochemistry, molecular biology). Presumably, compensating factors that are active during early chloroplast biogenesis would permit the desaturation reactions of carotenogenesis to occur in a PTOX-independent manner under high light conditions, e.g., by other oxidases or by PTOX-independent pathways of $\mathrm{PQH}_{2}$ re-oxidation coupled to PDS (discussed earlier). Shahbazi et al. (2007) provided early support for this idea on the basis of experiments showing that phytoene did not accumulate following exposure of mature green leaf or fruit tissues of the ghost mutant to high light: carotenoid synthesis occurred normally in the absence of PTOX. Later in chloroplast development we assume that compensating factors include elements of the PETC downstream of the PQ pool that are capable of oxidizing plastoquinol, thus obviating the need for PTOX.

In this context, it might be noted that early studies showing an $\mathrm{O}_{2}$ requirement for PDS activity were based on in vitro systems from non-photosynthetic chromoplasts (Beyer et al., 1989; Mayer et al., 1990). Interestingly, an "oxidoreductase fraction" was proposed to act as an intermediate between PDS and $\mathrm{O}_{2}$ in these systems (Beyer et al., 1989). We suggest that this $\mathrm{O}_{2}$ requirement reflects a need for PTOX activity in the absence of compensating factors, such as photosynthetic electron transport. This would be consistent with the general conclusion that PTOX is required for carotenogenesis during chloroplast biogenesis and stress, but not during steady state photosynthesis (Rosso et al., 2006, 2009).

\section{AOX2 and AOX1a suppressors}

To identify im suppressors, we have conducted EMS and activation-tagging mutagenesis of $\mathrm{im}$ and screened for mutant plants with a non-variegated phenotype. Molecular characterization of one suppressor line (designated ATG791) has been reported (Fu et al., 2012). This line is all-green and, surprisingly, carries an activation-tagged (overexpressed) version of mitochondrial AOX2. Arabidopsis contains five members of the AOX gene family, all of which are thought to be exclusively mitochondrial in location (AOX1a-d and AOX2); AOX2 is a low abundance, seedspecific member of this family (Saisho et al., 1997, 2001; Clifton et al., 2006; Winter et al., 2007; Polidoros et al., 2009).

We found that AOX2 is targeted to chloroplast thylakoids of ATG791, where its activity replaces that of PTOX in the desaturation steps of carotenogenesis, i.e., it rescues the phytoeneaccumulation defect of im, restoring carotenoid biosynthesis to normal. This restoration is accompanied by a normalization of excitation pressures, suggesting that the two processes are linked (Fu et al., 2012). It is therefore likely that elevated carotenoids in the suppressor lines are directly responsible for rescue of the variegation phenotype, and hence for recovery of the capacity to undergo normal chloroplast biogenesis. Results similar to ATG791 were obtained when AOX2 was overexpressed using the CaMV $35 \mathrm{~S}$ promoter, or when $A O X 1 a$ - the most highly and ubiquitously expressed member of the Arabidopsis AOX gene family, was re-engineered to target the plastid; overexpressed AOX1a is found exclusively in mitochondria (Clifton et al., 2006; Winter et al., 2007; Polidoros et al., 2009). In both cases, the im-induced defects in phytoene-accumulation and chloroplast biogenesis were reversed.

Further experiments with the AOX2 and AOX1a overexpression lines showed that chloroplast-localized AOX2, but not AOX1a, formed monomers and dimers in the thylakoid membrane, reminiscent of AOX regulation in mitochondrial inner membranes. In addition, both proteins accumulated as higher molecular weight complexes in thylakoids, though these complexes differed in size 
and number between the two lines. Interestingly, photosynthetic activities, as monitored by chlorophyll fluorescence, were not generally perturbed in the overexpression lines, nor was growth altered. This suggests that the presence of AOX1a and AOX2 complexes in thylakoids does not significantly perturb steady state photosynthesis, at least under non-stress conditions. Our operating assumption is that the suppressor lines define novel electron transport paths in the chloroplast.

AOX2 has previously been localized to mitochondria (Saisho et al., 2001), and because it was imported into chloroplasts using its own transit peptide in the $A O X 2$ overexpression lines, as well as in transient expression assays, our current working hypothesis is that AOX2 is a dual-targeted protein that functions in plastids to supplement PTOX activity during the early events of chloroplast biogenesis. Although the idea that AOX2 is normally an im-compensating activity needs further confirmation, the ability of AOX1a to substitute for PTOX in the correct physiological and developmental contexts is a dramatic example of the capacity of a mitochondrial protein to replace the function of a chloroplast protein, and illustrates the plasticity of the photosynthetic apparatus. This is all the more remarkable given the striking differences in regulation, substrate-specificity (ubiquinol in mitochondria versus plastoquinol in plastids) and phylogenetic distance between AOX and PTOX (McDonald et al., 2011; Fu et al., 2012). It will therefore be of great interest to explore further how chloroplast AOX1a and AOX2 mediate redox reactions during photosynthesis.

\section{pgr5 and crr2-2 suppressors}

Shikanai's group has long been studying mechanisms of PSI cyclic electron transport, a process that contributes to $\Delta \mathrm{pH}$ formation across the thylakoid, but not to $\mathrm{NAD}(\mathrm{P}) \mathrm{H}$ accumulation, thus providing a way of manipulating $\mathrm{ATP} / \mathrm{NAD}(\mathrm{P}) \mathrm{H}$ ratios (Shikanai, 2007). Okegawa et al. (2010) reported that two mutants perturbed in this process - crr2-2 (chlororespiratory reduction) (Hashimoto et al., 2003) and pgr5 (proton gradient regulation 5) (Munekage et al., 2002) are able to suppress im variegation. The pgr5 mutant is defective in electron transport, has a reduced $\Delta \mathrm{pH}$, an over-reduced stroma (a decreased ATP/NADPH), an oxidized $\mathrm{P}^{-}{ }^{+}$, and a loss of qE (Munekage et al., 2002; DalCorso et al., 2008; Okegawa et al., 2008). The crr2-2 mutant, on the other hand, is defective in NDH activity due to aberrant expression of the plastid $n d h B$ gene (Hashimoto et al., 2003); it has

\section{REFERENCES}

Adam, Z., Charuvi, D., Tsabari, O., Knopf, R. R., and Reich, Z. (2011). Biogenesis of thylakoid networks in angiosperms: knowns and unknowns. Plant Mol. Biol. 76, 221-234.

Al-Babili, S., Lintig, J. V., Haubruck, H., and Beyer, P. (1996). A novel, soluble form of phytoene desaturase from Narcissus pseudonarcissus chromoplasts is Hsp70-complexed and competent for flavination, membrane association and enzymatic activation. Plant J. 9, 601-612.

Allahverdiyeva, Y., Mamedov, F., Mäenpää, P., Vass, I., and Aro, E. M. (2005).

photosynthetic characteristics similar to pgr5 (Okegawa et al., 2010). Neither of these mutants has a chloroplast development phenotype (Munekage et al., 2004).

Okegawa et al. (2010) assumed that crr2-2 and pgr5 define separate pathways of PSI cyclic electron transport, and given this assumption, the goal of their experiments was to examine genetic interactions between these pathways and PTOX, all of which share the PQ pool. The observation that loss of either one of these pathways (in crr2-2 or pgr5) or both (in crr2-2/pgr5) is able to suppress $i m$ variegation can be explained by reduced excitation pressures during early chloroplast development, due to a decreased flux of electrons into the PQ pool. The converse suppression by im of the photosynthetic defects in $c r r 2-2$ and pgr5 is more problematic to explain, but might be due to altered ATP/NADPH ratios and activation of the water-water cycle in an im background (Okegawa et al., 2010).

One factor complicating an understanding of the mechanism of $i m$ suppression in these studies is that considerable controversy surrounds the function of PGR5 (reviewed in Kramer and Evans, 2011). The only agreement seems to be that PGR5 plays a role in fluctuating light conditions (Tikkanen et al., 2010). Although further studies will be needed to define the interactions between PTOX, PGR5, and NDH, it is clear from the present data that interactions occur between these three components very early in chloroplast development. All three are also present in etioplasts (Aluru et al., 2001; Long et al., 2008; Peng et al., 2008), perhaps to help poise the PQ pool for photosynthesis.

In summary, suppressor analysis holds great promise as a tool to understand the function of PTOX and mechanisms of compensation that occur in its absence. The data in this paper high light the significance of $i m$ suppressor analysis for understanding pathways and interactions that mediate early chloroplast development, and they include the identification of novel proteins (AOX2) and pathways (PSI cyclic electron transport).

\section{ACKNOWLEDGMENTS}

We extend our thanks to Margaret Carter of the Iowa State Image Analysis Facility. Additionally, we thank Dr. Harry Horner and Tracey Pepper of the Iowa State Microscopy and NanoImaging Facility. This work was supported by funding to Steve Rodermel from the Chemical Sciences, Geosciences, and Biosciences Division, U.S. Department of Energy (DE-FG02-94ER20147).

chloroplast terminal oxidase. Photosyn. Res. 91, 11-23.

tron transport in the absence of terminal electron acceptors: characterization of the $r b c L$ deletion mutant of tobacco. Biochim. Biophys. Acta 1709, 69-83.

Aluru, M. R., Bae, H., Wu, D., and Rodermel, S. R. (2001). The Arabidopsis immutans mutation affects plastid differentiation and the morphogenesis of white and green sectors in variegated plants. Plant Physiol. 127, 67-77.

Aluru, M. R., Stessman, D., Spalding, M. H., and Rodermel, S. R. (2007). Alterations in photosynthesis in Arabidopsis lacking IMMUTANS, a luru, M. R., Zola, J., Foudree, A., and Rodermel, S. R. (2009). Chloroplast photooxidation-induced transcriptome reprogramming in Arabidopsis immutans white leaf sectors. Plant Physiol. 150, 904-923.

Aro, E. M., Virgin, I., and Andersson, B. (1993). Photoinhibition of photosystem II: inactivation, protein damage and turnover. Biochim. Biophys. Acta 1143, 113-134.

Baena-González, E., Allahverdiyeva, Y., Svab, Z., Maliga, P., Josse, E. M., Kuntz, M., et al. (2003). Deletion of the tobacco plastid $p s b A$ gene triggers an upregulation of the thylakoid-associated $\mathrm{NAD}(\mathrm{P}) \mathrm{H}$ dehydrogenase complex and the plastid terminal oxidase (PTOX). Plant J. 35, 704-716.

Bailey, S., Melis, A., Mackey, K. R. M., Cardol, P., Finazzi, G., van Dijken G., et al. (2008). Alternative photosynthetic electron flow to oxygen in marine Synechococcus. Biochim. Biophys. Acta 1777, 269-276.

Barr, J., White, W. S., Chen, L., Bae, H., and Rodermel, S. (2004). The GHOST terminal oxidase regulates developmental programming in tomato fruit. Plant Cell Environ. 27, 840-852. 
Berthold, D. A., Andersson, M. E., and Nordlund, P. (2000). New insight into the structure and function of the alternative oxidase. Biochim. Biophys. Acta 1460, 241-254.

Beyer, P., Mayer, M., and Kleinig, $\mathrm{H}$. (1989). Molecular oxygen and the state of geometric isomerism of intermediates are essential in the carotene desaturation and cyclization reactions in daffodil chromoplasts. Eur. J. Biochem. 184, 141-150.

Blomqvist, L. A., Ryberg, M., and Sundqvist, C. (2008). Proteomic analysis of highly purified prolamellar bodies reveals their significance in chloroplast development. Photosyn. Res. 96, 37-50.

Buchel, C., and Garab, G. (1995). Evidence for the operation of a cyanidesensitive oxidase in chlororespiration in the thylakoids of the chlorophyll $c$-containing alga Pleurochloris meiringensis (Xanthophyceae). Planta 197, 69-75.

Carol, P., Stevenson, D., Bisanz, C., Breitenbach, J., Sandmann, G., Mache, R., et al. (1999). Mutations in the Arabidopsis gene IMMUTANS cause a variegated phenotype by inactivating a chloroplast terminal oxidase associated with phytoene desaturation. Plant Cell 11, 57-68.

Casano, L. M., Zapata, J. M., Martin, M., and Sabater, B. (2000). Chlororespiration and poising of cyclic electron transport. J. Biol. Chem. 275, 942-948.

Clifton, R., Millar, A. H., and Whelan, J. (2006). Alternative oxidases in Arabidopsis: a comparative analysis of differential expression in the gene family provides new insights into function of nonphosphorylating bypasses. Biochim. Biophys. Acta 1757, 730-741.

DalCorso, G., Pesaresi, P., Masiero, S., Aseeva, E., Schunemann, D., Finazzi, G., et al. (2008). A complex containing PGRL1 and PGR5 is involved in the switch between linear and cyclic electron flow in Arabidopsis. Cell 132, 273-285.

DellaPenna, D., and Pogson, B. J. (2006). Vitamin synthesis in plants: tocopherols and carotenoids. Annu. Rev. Plant Biol. 57, 711-738.

Díaz, M., De Haro, V., Munoz, R., and Quiles, M. J. (2007). Chlororespiration is involved in the adaptation of Brassica plants to heat and high light intensity. Plant Cell Environ. 30, 1578-1585.

Dietz, K. J., Schreiber, U., and Heber, U. (1985). The relationship between the redox state of $\mathrm{Q}_{\mathrm{A}}$ and photosynthesis in leaves at various carbon-dioxide, oxygen and light regimes. Planta 166, 219-226.

Formighieri, C., Ceol, M., Bonente, G., Rochaix, J. D., and Bassi, R. (2012). Retrograde signaling and photoprotection in a gun 4 mutant of Chlamydomonas reinhardtii. Mol. Plant. doi:10.1093/mp/sss051

Foyer, C. H., Neukermans, J., Queval, G., Noctor, G., and Harbinson, J. (2012). Photosynthetic control of electron transport and the regulation of gene expression. J. Exp. Bot. 63, 1637-1661.

Fu, A., Liu, H., Yu, F., Kambakam, S., Luan, S., and Rodermel, S. (2012). Alternative oxidases (AOX1a and AOX2) can functionally substitute for plastid terminal oxidase in Arabidopsis chloroplasts. Plant Cell 24, 1579-1595.

Giraud, E., Ho, L. H., Clifton, R., Carroll, A., Estavillo, G., Tan, Y. F., et al. (2008). The absence of ALTERNATIVE OXIDASEla in Arabidopsis results in acute sensitivity to combined light and drought stress. Plant Physiol. 147, 595-610.

Gray, G. R., Chauvin, L. P., Sarhan, F., and Huner, N. P. A. (1997). Cold acclimation and freezing tolerance. A complex interaction of light and temperature. Plant Physiol. 114, 467-474.

Hashimoto, M., Endo, T., Peltier, G., Tasaka, M., and Shikanai, T. (2003). A nucleus-encoded factor, CRR2, is essential for the expression of chloroplast $n d h B$ in Arabidopsis. Plant J. 36, 541-549.

Houille-Vernes, L., Rappaport, F., Wollman, F.-A., Alric, J., and Johnson, X. (2011). Plastid terminal oxidase 2 (PTOX2) is the major oxidase involved in chlororespiration in Chlamydomonas. Proc. Natl. Acad. Sci. U.S.A. 108, 20820-20825.

Hugueney, P., Römer, S., Kuntz, M., and Camara, B. (1992). Characterization and molecular cloning of a flavoprotein catalyzing the synthesis of phytofluene, and zeta-carotene in Capsicum chromoplasts. Eur. J. Biochem. 209, 399-407.

Hüner, N. P. A., Öquist, G., and Sarhan, F. (1998). Energy balance and acclimation to light and cold. Trends Plant Sci. 3, 224-230.

Ivanov, A. G., Rosso, D., Savitch, L. V., Stachula, P., Rosembert, M., Oquist, G., et al. (2012). Implications of alternative electron sinks in increased resistance of PSII and PSI photochemistry to high light stress in cold-acclimated Arabidopsis thaliana. Photosyn. Res. 113, 191-206.
Joët, T., Genty, B., Josse, E. M., Kuntz, M., Cournac, L., and Peltier, G. (2002). Involvement of a plastid terminal oxidase in plastoquinone oxidation as evidenced by expression of the Arabidopsis thaliana enzyme in tobacco. J. Biol. Chem. 277, 31623-31630.

Josse, E. M., Alcaraz, J.-P., Labouré, A. M., and Kuntz, M. (2003). In vitro characterization of a plastid terminal oxidase (PTOX). Eur. J. Biochem. 270, 3787-3794.

Josse, E. M., Simkin, A. J., Gaffé, J., Labouré, A. M., Kuntz, M., and Carol, P. (2000). A plastid terminal oxidase associated with carotenoid desaturation during chromoplast differentiation. Plant Physiol. 123, 1427-1436.

Joyard, J., Ferro, M., Masselon, C., Seigneurin-Berny, D., Salvi, D., Garin, J., et al. (2009). Chloroplast proteomics and the compartmentation of plastidial isoprenoid biosynthetic pathways. Mol. Plant 2, 1154-1180.

Kanervo, E., Singh, M., Suorsa, M., Paakkarinen, V., Aro, E., Battchikova, N., et al. (2008). Expression of protein complexes and individual proteins upon transition of etioplasts to chloroplasts in pea (Pisum sativum). Plant Cell Physiol. 49, 396-410.

Khorobrykh, S. A., and Ivanov, B. N. (2002). Oxygen reduction in a plastoquinone pool of isolated pea thylakoids. Photosyn. Res. 71, 209-219.

Kramer, D. M., and Evans, J. R. (2011). The importance of energy balance in improving photosynthetic productivity. Plant Physiol. 155, 70-78.

Krause, G. H., and Weis, E. (1991). Chlorophyll fluorescence and photosynthesis: the basics. Annu. Rev. Plant Physiol. Plant Mol. Biol. 42, 313-349.

Lajko, F., Kadioglu, A., Borbely, G., and Garab, G. (1997). Competition between the photosynthetic and the (chloro)respiratory electron transport chains in cyanobacteria, green algae and higher plants. Effect of heat stress. Photosynthetica 33, 217-226.

Laureau, C., Bligny, R., and Streb, P. (2011). The significance of glutathione for photoprotection at contrasting temperatures in the alpine plant species Soldanella alpina and Ranunculus glacialis. Physiol. Plant 143, 246-260.

Lennon, A. M., Prommeenate, P., and Nixon, P. J. (2003). Location, expression and orientation of the putative chlororespiratory enzymes, $\mathrm{Ndh}$ and IMMUTANS, in higher-plant plastids. Planta 218, 254-260.

Liu, X., Yu, F., and Rodermel, S. (2010). Arabidopsis chloroplast $\mathrm{FtsH}$, var2 and suppressors of var2 leaf variegation: a review. J. Integr. Plant Biol. 52, 750-761.

Long, T. A., Okegawa, Y., Shikanai, T., Schmidt, G. W., and Covert, S. F. (2008). Conserved role of proton gradient regulation 5 in the regulation of PSI cyclic electron transport. Planta 228, 907-918.

López-Juez, E. (2009). Steering the solar panel: plastids influence development. New Phytol. 182, 287-290.

Mansfield, S. G., and Briarty, L. G. (1996). The dynamics of seedling and cotyledon cell development in Arabidopsis thaliana during reserve mobilization. Int. J. Plant Sci. 157, 280-295.

Mayer, M. P., Beyer, P., and Kleinig, H. (1990). Quinone compounds are able to replace molecular oxygen as terminal electron acceptor in phytoene desaturation in chromoplasts of Narcissus pseudonarcissus L. Eur. J. Biochem. 191, 359-363.

Mayer, M. P., Nievelstein, V., and Beyer, P. (1992). Purification and characterization of a NADPH dependent oxidoreductase from chromoplasts of Narcissus pseudonarcissus: a redox-mediator possibly involved in carotene desaturation. Plant Physiol. Biochem. 30, 389-398.

Mayfield, S. P., and Taylor, W. C. (2005). Carotenoid-deficient maize seedlings fail to accumulate lightharvesting chlorophyll $a / b$ binding protein (LHCP) mRNA. Eur. J. Biochem. 144, 79-84.

McDonald, A. E. (2008). Alternative oxidase: an inter-kingdom perspective on the function and regulation of this broadly distributed "cyanideresistant" terminal oxidase. Funct. Plant Biol. 35, 535-552.

McDonald, A. E., Ivanov, A. G., Bode, R., Maxwell, D. P., Rodermel, S. R., and Hüner, N. P. A. (2011). Flexibility in photosynthetic electron transport: the physiological role of plastoquinol terminal oxidase (PTOX). Biochim. Biophys. Acta 1807, 954-967.

Meehan, L., Harkins, K., Chory, J., and Rodermel, S. (1996). Lhcb transcription is coordinated with cell size and chlorophyll accumulation. Studies on fluorescence-activated, cell-sorter-purified single cells from wild-type and immutans Arabidopsis thaliana. Plant Physiol. 112, 953-963.

Møller, I. M., Jensen, P. E., and Hansson, A. (2007). Oxidative modifications 
to cellular components in plants. Annu. Rev. Plant Biol. 58, 459-481.

Moseley, J. L., Chang, C. W., and Grossman, A. R. (2006). Genome-based approaches to understanding phosphorus deprivation responses and PSR1 control in Chlamydomonas reinhardtii. Eukaryot. Cell 5, 26-44.

Mullet, J. E. (1988). Chloroplast development and gene expression. Annu. Rev. Plant Physiol. Plant Mol. Biol. 39, 475-502.

Munekage, Y., Hashimoto, M., Miyake, C., Tomizawa, K., Endo, T., Tasaka, M., et al. (2004). Cyclic electron flow around photosystem I is essential for photosynthesis. Nature 429, 579-582.

Munekage, Y., Hojo, M., Meurer, J., Endo, T., Tasaka, M., and Shikanai, T. (2002). PGR5 is involved in cyclic electron flow around photosystem I and is essential for photoprotection in Arabidopsis. Cell 110, 361-371.

Ngaki, M. N., Louie, G. V., Philippe, R. N., Manning, G., Pojer, F., Bowman, M. E., et al. (2012). Evolution of the chalcone isomerase fold from fatty acid-binding to stereospecific enzyme. Nature 485, 53-533.

Nievelstein, V., Vandekerckhove, J., Tadros, M. H., von Lintig, J., Nitschke, W., and Beyer, P. (1995). Carotene desaturation is linked to a respiratory redox pathway in Narcissus pseudonarcissus chromoplast membranes: involvement of a 23-kDa oxygen-evolving-complexlike protein. Eur. J. Biochem. 233, 864-872.

Niyogi, K. K. (2000). Safety valves for photosynthesis. Curr. Opin. Plant Biol. 3, 455-460.

Norris, S. R., Barrette, T. R., and DellaPenna, D. (1995). Genetic dissection of carotenoid synthesis in Arabidopsis defines plastoquinone as an essential component of phytoene desaturation. Plant Cell 7, 2139-2149.

Oelmüller, R. (1989). Photooxidative destruction of chloroplasts and its effect on nuclear gene expression and extraplastidic enzyme levels. Photochem. Photobiol. 49, 229-239.

Okegawa, Y., Kagawa, Y., Kobayashi, Y., and Shikanai, T. (2008). Characterization of factors affecting the activity of photosystem I cyclic electron transport in chloroplasts. Plant Cell Physiol. 49, 825-834.

Okegawa, Y., Kobayashi, Y., and Shikanai, T. (2010). Physiological links among alternative electron transport pathways that reduce and oxidize plastoquinone in Arabidopsis. Plant J. 63, 458-468.

Ort, D. R., and Baker, N. R. (2002). A photoprotective role for $\mathrm{O}_{2}$ as an alternative electron sink in photosynthesis? Curr. Opin. Plant Biol. 5, 193-198.

Peltier, G., and Cournac, L. (2002). Chlororespiration. Annu. Rev. Plant Biol. 53, 523-550.

Peng, L., Fukao, Y., Fujiwara, M., and Shikanai, T. (2012). Multistep assembly of chloroplast $\mathrm{NADH}$ dehydrogenase-like subcomplex A requires several nucleus-encoded proteins, including CRR41 and CRR42, in Arabidopsis. Plant Cell 24, 202-214.

Peng, L., Shimizu, H., and Shikanai, T. (2008). The chloroplast NAD(P)H dehydrogenase complex interacts with photosystem I in Arabidopsis. J. Biol. Chem. 283, 34873-34879.

Polidoros, A. N., Mylona, P. V., and Arnholdt-Schmitt, B. (2009). AOX gene structure, transcript variation and expression in plants. Physiol. Plant 137, 342-354.

Possingham, J. V., and Lawrence, M. E. (1983). Controls to plastid division. Int. Rev. Cytol. 84, $1-56$.

Pyke, K., and López-Juez, E. (1999). Cellular differentiation and leaf morphogenesis in Arabidopsis. CRC Crit. Rev. Plant Sci. 18, 527-546.

Pyke, K. A., Marrison, J. L., and Leech, R. M. (1991). Temporal and spatial development of the cells of the expanding first leaf of Arabidopsis thaliana (L.) Heynh. J. Exp. Bot. 42, 1407-1416.

Quiles, M. J. (2006). Stimulation of chlororespiration by heat and high light intensity in oat plants. Plant Cell Environ. 29, 1463-1470.

Rédei, G. P. (1963). Somatic instability caused by a cysteine-sensitive gene in Arabidopsis. Science 139, 767-769.

Rédei, G. P. (1967). Biochemical aspects of a genetically determined variegation in Arabidopsis. Genetics 56, 431-443.

Rizhsky, L., Hallak-Herr, E., Van Breusegem, F., Rachmilevitch, S., Barr, J. E., Rodermel, S., et al. (2002). Double antisense plants lacking ascorbate peroxidase and catalase are less sensitive to oxidative stress than single antisense plants lacking ascorbate peroxidase or catalase. Plant J. 32, 329-342.

Röbbelen, G. (1968). Genbedingte Rotlicht-Empfindlichkeit der Chloroplastendifferenzierung bei Arabidopsis. Planta 80, 237-254.
Robertson, E. J., Pyke, K. A., and Leech, R. M. (1995). arc6, An extreme chloroplast division mutant of Arabidopsis, also alters proplastid proliferation and morphology in shoot and root apices. J. Cell Sci. 108, 2937-2944.

Rochaix, J. D. (2011). Regulation of photosynthetic electron transport. Biochim. Biophys. Acta 1807, 878-886.

Rodermel, S. R. (2001). Pathways of plastid-to-nucleus signaling. Trends Plant Sci. 6, 471-478.

Rodrigo, M. J., Marcos, J. F., and Zacarias, L. (2004). Biochemical and molecular analysis of carotenoid biosynthesis in flavedo of orange (Citrus sinensis L.) during fruit development and maturation. $J$ Agric. Food Chem. 52, 6724-6731.

Rodríguez-Villalón, A., Gas, E., and Rodríguez-Concepción, M. (2009). Phytoene synthase activity controls the biosynthesis of carotenoids and the supply of their metabolic precursors in dark-grown Arabidopsis seedlings. Plant J. 60 424-435.

Rosso, D., Bode, R., Li, W., Krol, M., Saccon, D., Wang, S., et al. (2009). Photosynthetic redox imbalance governs leaf sectoring in the Arabidopsis thaliana variegation mutants immutans, spotty, var1 and var2. Plant Cell 21, 3473-3492.

Rosso, D., Ivanov, A. G., Fu, A., GeislerLee, J., Hendrickson, L., Geisler, M., et al. (2006). IMMUTANS does not act as a stress-induced safety valve in the protection of the photosynthetic apparatus of Arabidopsis during steady-state photosynthesis. Plant Physiol. 142, 574-585.

Ruckle, M. E., and Larkin, R. M. (2009). Plastid signals that affect photomorphogenesis in Arabidopsis thaliana are dependent on GENOMES UNCOUPLED 1 and cryptochrome 1. New Phytol. 182, 367-379.

Rumeau, D., Peltier, G., and Cournac, L. (2007). Chlororespiration and cyclic electron flow around PSI during photosynthesis and plant stress response. Plant Cell Environ. 30, 1041-1051.

Saisho, D., Nakazono, M., Lee, K. H., Tsutsumi, N., Akita, S., and Hirai, A. (2001). The gene for alternative oxidase-2 (AOX2) from Arabidopsis thaliana consists of five exons unlike other AOX genes and is transcribed at an early stage during germination. Genes Genet. Syst. 76, 89-97.

Saisho, D., Nambara, E., Naito, S., Tsutsumi, N., Hirai, A., and Nakazono, M. (1997). Characterization of the gene family for alternative oxidase from Arabidopsis thaliana. Plant Mol. Biol. 35, 585-596.

Savitch, L. V., Ivanov, A. G., Krol, M. K., Sprott, D. P., Oquist, G., and Huner, N. P. A. (2010). Regulation of energy partitioning and alternative electron transport pathways during cold acclimation of Lodgepole pine is oxygen dependent. Plant Cell Physiol. 51, 1555-1570.

Savitch, L. V., Massacci, A., Gray, G. R., and Huner, N. P. A. (2000). Acclimation to low temperature or high light mitigates sensitivity to photoinhibition: roles of the Calvin cycle and the Mehler reaction. Aust. J. Plant Physiol. 27, 253-264.

Schulz, A., Ort, O., Beyer, P., and Kleinig, H. (1993). SC-0051, a 2-benzoylcyclohexane-1,3-dione bleaching herbicide, is a potent inhibitor of the enzyme p-hydroxyphenylpyruvate dioxygenase. FEBS Lett. 318, 162-166.

Shahbazi, M., Gilbert, M., Labouré, A. M., and Kuntz, M. (2007). Dual role of the plastid terminal oxidase in tomato. Plant Physiol. 145, 691-702.

Shikanai, T. (2007). Cyclic electron transport around photosystem I: genetic approaches. Annu. Rev. Plant Biol. 58, 199-217.

Smith, W. K., Vogelmann, T. C., DeLucia, E. H., Bell, D. T., and Shepherd, K. A. (1997). Leaf form and photosynthesis: do leaf structure and orientation interact to regulate internal light and carbon dioxide? Bioscience 47, 785-793.

Solymosi, K., Morandi, D., Bóka, K., Böddi, B., and Schoefs, B. (2012). High biological variability of plastids, photosynthetic pigments and pigment forms of leaf primordia in buds. Planta 235, 1035-1049.

Solymosi, K., and Schoefs, B. (2010). Etioplast and etio-chloroplast formation under natural conditions: the dark side of chlorophyll biosynthesis in angiosperms. Photosyn. Res. 105, 143-166.

Stepien, P., and Johnson, G. N. (2009). Contrasting responses of photosynthesis to salt stress in the glycophyte Arabidopsis and the halophyte Thellungiella: role of the plastid terminal oxidase as an alternative electron sink. Plant Physiol. 149, 1154-1165.

Streb, P., Josse, E. M., Gallouët, E., Baptist, F., Kuntz, M., and Cornic, G. (2005). Evidence for alternative electron sinks to photosynthetic carbon assimilation in the high mountain plant species Ranunculus glacialis. Plant Cell Environ. 28, 1123-1135. 
Tikkanen, M., Grieco, M., Kangasjarvi, S., and Aro, E. M. (2010). Thylakoid protein phosphorylation in higher plant chloroplasts optimizes electron transfer under fluctuating light. Plant Physiol. 152, 723-735.

Tilney-Bassett, R. A. E. (1975). "Genetics of variegated plants," in Genetics and Biogenesis of Mitochondria and Chloroplasts, eds C. W. Birky, P. S. Perlman, and T. J. Byers (Columbus, $\mathrm{OH}$ : Ohio State University Press), 268-308.

Trouillard, M., Shahbazi, M., Moyet, L., Rappaport, F., Joliot, P., Kuntz, M., et al. (2012). Kinetic properties and physiological role of the plastoquinone terminal oxidase (PTOX) in a vascular plant. Biochim. Biophys. Acta 1817, 2140-2148.

Van Lijsebettens, M., and Clarke, J. (1998). Leaf development in Arabidopsis. Plant Physiol. Biochem. 36, 47-60.

Velez-Ramirez, A. I., van Ieperen, W., Vreugdenhil, D., and Millenaar, F. F. (2011). Plants under continuous light. Trends Plant Sci. 16, 310-318. von Zychlinski, A., Kleffmann, T., Krishnamurthy, N., Sjolander, K. Baginsky, S., and Gruissem, W. (2005). Proteome analysis of the rice etioplast. Mol. Cell. Proteomics 4, 1072-1084.

Wang, J., Sommerfeld, M., and $\mathrm{Hu}$, Q. (2009). Occurrence and environmental stress responses of two plastid terminal oxidases in Haematococcus pluvialis (Chlorophyceae). Planta 230, 191-203.

Wetzel, C. M., Jiang, C. Z., Meehan, L. J., Voytas, D. F., and Rodermel, S. R. (1994). Nuclear-organelle interactions: the immutans variegation mutant of Arabidopsis is plastid autonomous and impaired in carotenoid biosynthesis. Plant J. 6, 161-175.

Wetzel, C. M., and Rodermel, S. (1998). Regulation of phytoene desaturase expression is independent of leaf pigment content in Arabidopsis thaliana. Plant Mol. Biol.37, 1045-1053.

Winter, D., Vinegar, B., Nahal, H., Ammar, R., Wilson, G. V., and
Provart, N. J. (2007). An "electronic fluorescent pictograph" browser for exploring and analyzing large-scale biological data sets. PLoS ONE 8, e718. doi:10.1371/journal.pone.0000718

Woo, N. S., Gordon, M. J., Graham, S. R., Rossel, J. B., Badger, M. R., and Pogson, B. J. (2011). A mutation in the purine biosynthetic enzyme ATASE2 impacts high light signalling and acclimation responses in green and chlorotic sectors of Arabidopsis leaves. Funct. Plant Biol. 38, 401-419.

Wu, D., Wright, D. A., Wetzel, C., Voytas, D. F., and Rodermel, S. (1999). The IMMUTANS variegation locus of Arabidopsis defines a mitochondrial alternative oxidase homolog that functions during early chloroplast biogenesis. Plant Cell 11, 43-55.

Yu, F., Fu, A., Aluru, M., Park, S., Xu, Y., Liu, H., et al. (2007). Variegation mutants and mechanisms of chloroplast biogenesis. Plant Cell Environ. 30, 350-365.

Conflict of Interest Statement: The authors declare that the research was conducted in the absence of any commercial or financial relationships that could be construed as a potential conflict of interest.

Received: 21 September 2012; accepted: 06 November 2012; published online: 27 November 2012.

Citation: Foudree A, Putarjunan A, Kambakam S, Nolan T, Fussell J, Pogorelko $G$ and Rodermel S (2012) The mechanism of variegation in immutans provides insight into chloroplast biogenesis. Front. Plant Sci. 3:260. doi: 10.3389/fpls.2012.00260

This article was submitted to Frontiers in Plant Physiology, a specialty of Frontiers in Plant Science.

Copyright (c) 2012 Foudree, Putarjunan, Kambakam, Nolan, Fussell, Pogorelko and Rodermel. This is an open-access article distributed under the terms of the Creative Commons Attribution License, which permits use, distribution and reproduction in other forums, provided the original authors and source are credited and subject to any copyright notices concerning any third-party graphics etc. 